\title{
Recognition, assessment and management of hypoglycaemia in childhood
}

\author{
Arunabha Ghosh, ${ }^{1}$ Indraneel Banerjee, ${ }^{2}$ Andrew A M Morris ${ }^{1}$
}

'Paediatric Inherited Metabolic Disease, Manchester Centre for Genomic Medicine, St Mary's Hospital, Manchester, UK ${ }^{2}$ Paediatric Endocrinology, Royal Manchester Children's Hospital, Manchester, UK

\section{Correspondence to}

Dr Arunabha Ghosh, Paediatric Inherited Metabolic Disease,

Manchester Centre for

Genomic Medicine, St Mary's

Hospital, Manchester M13

9WL, UK;

arunabha.ghosh@cmft.nhs.uk

Received 12 August 2015 Revised 16 November 2015 Accepted 18 November 2015 Published Online First 30 December 2015

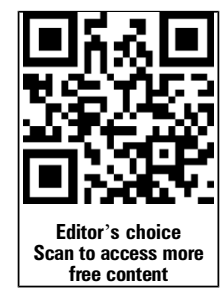

CrossMark

To cite: Ghosh A,

Banerjee I, Morris AAM.

Arch Dis Child

2016;101:575-580.

\section{ABSTRACT}

Hypoglycaemia is frequent in children and prompt management is required to prevent brain injury. In this article we will consider hypoglycaemia in children after the neonatal period. The most common causes are diabetes mellitus and idiopathic ketotic hypoglycaemia (IKH) but a number of endocrine disorders and inborn errors of metabolism (IEMs) need to be excluded. Elucidation of the diagnosis relies primarily on investigations during a hypoglycaemic episode but may also involve biochemical tests between episodes, dynamic endocrine tests and molecular genetics. Specific treatment such as cortisol replacement and pancreatic surgery may be required for endocrine causes of hypoglycaemia, such as adrenal insufficiency and congenital hyperinsulinism. In contrast, in IKH and most IEMs, hypoglycaemia is prevented by limiting the duration of fasting and maintaining a high glucose intake during illnesses.

\section{INTRODUCTION}

Glucose is the preferred fuel for the brain other than during periods of prolonged starvation, and hypoglycaemia therefore has the capacity to cause brain injury. Potential sequelae include neurocognitive defects, memory deficits, aphasia and hemiparesis. ${ }^{1}$ Accurate recognition and urgent treatment of hypoglycaemic episodes are, therefore, crucial. Perinatal transitional hypoglycaemia has been considered in several recent articles ${ }^{2}{ }^{3}$; this review will focus on hypoglycaemia in children and infants beyond the neonatal period.

\section{Glucose homeostasis}

The brain has limited glycogen stores and capacity for gluconeogenesis and therefore requires a continuous glucose supply. Insulin and the counterregulatory hormones (principally glucagon) tightly regulate glucose homeostasis.

After a meal, blood glucose levels rise and pancreatic $\beta$-cells secrete insulin in response. Insulin increases glucose uptake in skeletal muscle and adipose tissue by increasing GLUT4 receptor expression. Insulin also promotes glycogen synthesis and inhibits both glycogenolysis and hepatic gluconeogenesis. ${ }^{4}$ Glucose that is not immediately used is stored as glycogen in liver and muscle or converted to lipid.

Various counter-regulatory mechanisms normally maintain glucose homeostasis in the fasted state (figure 1). Insulin secretion is suppressed, while glucagon is secreted, promoting glucose production from glycogen in the liver. In healthy children, after $12-16 \mathrm{~h}$ of fasting, glycogen stores become depleted; glucose is then produced by hepatic gluconeogenesis, primarily from amino acids and glycerol. ${ }^{5}$ Glucagon, adrenaline, cortisol and growth hormone promote gluconeogenesis and activate lipolysis, increasing the supply of fatty acids as an energy source and reducing glucose consumption. Fatty acid oxidation also provides acetylcoenzyme A (CoA) for ketogenesis. After prolonged fasting (in excess of $24 \mathrm{~h}$ ), ketone bodies become the primary source of energy for the brain. ${ }^{6}$

\section{RECOGNITION OF HYPOGLYCAEMIA}

Prompt detection of hypoglycaemia requires measurement of the blood glucose concentration whenever there are relevant symptoms and also routinely when there is a high risk, for example, in severely ill or diabetic children.

\section{Clinical presentation of hypoglycaemia}

The counter-regulatory responses to hypoglycaemia are triggered at plasma glucose concentrations just below the normal physiological range (approximately $3.8 \mathrm{mmol} / \mathrm{L}$ ). If these fail, a sympathoadrenal response develops, at plasma glucose concentrations below approximately $3.0 \mathrm{mmol} / \mathrm{L}$. Early manifestations of hypoglycaemia are therefore autonomic in nature, such as pallor, anxiety, sweating, weakness, tremor, tachypnoea, nausea and vomiting ${ }^{8}$ and in older children there may be a behavioural response (eg, to search for food). If hypoglycaemia worsens, neuroglycopenic symptoms supervene, leading to irritability, confusion, slurred speech, headache and eventually seizures and coma. 89

The clinical features of hypoglycaemia are caused by failure to deliver glucose or alternative fuels to the brain. Ketone bodies are the principal alternative fuel and cross the blood-brain barrier on MCT1 transporters, which are upregulated in patients with chronically raised ketone body levels. ${ }^{10}$ Thus, hypoglycaemia may cause few symptoms in children on a ketogenic diet or adults after prolonged fasting. In fasted children, ketone bodies are less effective in preventing the symptoms of hypoglycaemia; this is probably because hypoglycaemia occurs earlier, before MCT1 expression has increased. The brain can also use lactate as a fuel if its concentration is chronically raised, as in glycogen storage disease type I (GSD I) prior to diagnosis. These infants often tolerate hypoglycaemia with relatively few symptoms ${ }^{11}$ but they become more vulnerable to the adverse effects of hypoglycaemia after treatment is commenced and lactate concentrations fall.

The manifestations of hypoglycaemia also depend on previous blood glucose concentrations. Patients with poorly controlled diabetes develop 


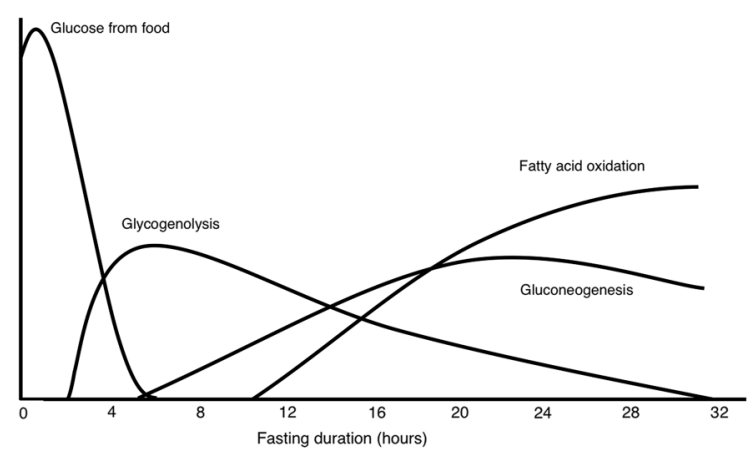

Figure 1 Energy sources during fasting.

symptoms of hypoglycaemia at higher glucose concentrations than normal subjects. ${ }^{12}$ Conversely, previous hypoglycaemia reduces the autonomic response and cognitive dysfunction during subsequent episodes of hypoglycaemia. ${ }^{13}$ As well as diminishing the counter-regulatory hormone response, this leads to 'hypoglycaemia unawareness'-the absence of warning symptoms, such as palpitations, tremor and hunger. Patients with diabetes are also much less likely to be awakened by nocturnal hypoglycaemia than normal individuals. ${ }^{14}$

\section{Definition of hypoglycaemia}

Clinical hypoglycaemia has been defined as 'a plasma glucose concentration low enough to cause symptoms and/or signs of impaired brain function'. ${ }^{15}$ This glucose concentration varies from one patient to another, depending on the availability of alternative fuels and previous blood glucose concentrations, as discussed above. Moreover, in neonates, a decrease in blood glucose is expected after delivery and is physiologically normal. ${ }^{2}$ Hypoglycaemia is, therefore, a spectrum and the blood glucose concentration should be interpreted together with the clinical history and concentrations of counter-regulatory hormones and intermediate metabolites. ${ }^{3}$

Point-of-care blood glucose tests have limited accuracy. Patients with symptoms suggesting hypoglycaemia should, therefore, be treated promptly regardless of the result. Treatment should also be given to asymptomatic children with a blood glucose concentration below $3.3 \mathrm{mmol} / \mathrm{L}$; maintenance of the blood glucose above $4 \mathrm{mmol} / \mathrm{L}$ is recommended in children with diabetes. Unless the diagnosis is known, every effort should be made to collect blood for analysis of counter-regulatory hormones and intermediate metabolites prior to treatment (see below, Laboratory investigation of hypoglycaemia).

The Pediatric Endocrine Society has recently published guidance on which patients warrant further investigation. This is recommended for children who fulfil Whipple's triad, that is, symptoms of hypoglycaemia, a low blood glucose concentration and resolution of the symptoms on correction of the glucose concentration. For children who are unable to communicate symptoms, they recommend evaluation of hypoglycaemia only if the plasma glucose concentration, confirmed by laboratory methods, is below $3.3 \mathrm{mmol} / \mathrm{L} .{ }^{15}$ We agree that investigation is unnecessary for glucose concentrations above this value. For lower plasma glucose concentrations, the clinical history, hormone and metabolite results should be reviewed. ${ }^{3}$ Further investigations will probably be appropriate if the plasma glucose was below $3.0 \mathrm{mmol} / \mathrm{L}$ but may not be needed for values between 3.0 and $3.3 \mathrm{mmol} / \mathrm{L}$.

\section{DIFFERENTIAL DIAGNOSIS OF HYPOGLYCAEMIA IN CHILDHOOD}

The most common causes of hypoglycaemia in children are diabetes and idiopathic ketotic hypoglycaemia (IKH, see below). Hypoglycaemia also occurs in other endocrine disorders, inborn errors of metabolism (IEMs), liver failure and secondary to poisoning or drug ingestion (box 1). Infections are often responsible for precipitating hypoglycaemia in patients with a disorder that puts them at risk and they can also cause hypoglycaemia without a predisposing condition. In most cases, hypoglycaemia is due to increased usage of glucose (hyperinsulinism, fatty acid oxidation disorders (FAODs), sepsis), decreased nutritional supply (gastroenteritis) or decreased endogenous production of glucose (adrenal insufficiency, IEMs, liver failure).

\section{Hyperinsulinaemic hypoglycaemia}

Exogenous insulin causes hypoglycaemia in diabetes mellitus and, on rare occasions, in children with induced illness. In contrast, excess endogenous insulin production by pancreatic $\beta$-cells causes hypoglycaemia in congenital hyperinsulinism (CHI). CHI usually presents in the neonatal period but may also present later in infancy and remains one of the most common causes of recurrent hypoglycaemia. The hypoglycaemia is usually severe, requiring glucose infusion rates above $8 \mathrm{mg} / \mathrm{kg} / \mathrm{min}$. Genetic defects are identified in half the cases, most commonly in the $\mathrm{K}^{+}$-ATP channel genes (ABCC8/KCNJ11). ${ }^{16} \mathrm{CHI}$ can be classified histologically as diffuse or focal. Focal $\mathrm{CHI}$ is identified by finding a paternal heterozygous mutation in $A B C C 8 / K C N J 11^{17}$ and a solitary focal area on ${ }^{18} \mathrm{~F}$-fluoro-L-dihydroxyphenylalanine $\left({ }^{18} \mathrm{~F}\right.$-DOPA) positron emission tomography (PET) scanning.

Hyperinsulinism also occurs in hyperinsulinism/hyperammonaemia syndrome (due to activating mutations of glutamate dehydrogenase) and in congenital disorders of glycosylation. The hypoglycaemia is often milder in these conditions and patients may present after infancy. Hyperinsulinism can also cause postprandial hypoglycaemia after gastric surgery, such as gastrostomy or Nissen fundoplication (dumping syndrome) ${ }^{18}$; excessive insulin secretion may be caused directly by carbohydrates rapidly entering the small intestine or indirectly through incretin activation. In older children with hyperinsulinaemic hypoglycaemia, the possibility of an insulinoma should be considered, either as an isolated entity or as part of multiple endocrine neoplasia type I syndrome.

\section{Other endocrine disorders}

Children with cortisol deficiency may present with hypoglycaemia. Causes include congenital adrenal hyperplasia, autoimmune adrenal insufficiency, X-linked adrenoleukodystrophy and hypopituitarism. Secondary cortisol deficiency occurs in a significant proportion of children with asthma on relatively large doses of inhaled steroids. Cortisol deficiency is usually accompanied by tiredness and lethargy, and some children present in extremis with an adrenal crisis. Growth hormone deficiency can also present with hypoglycaemia, either in association with cortisol deficiency or rarely in isolation.

\section{Defects of glycogen synthesis and degradation}

The GSDs are inherited defects of glycogen synthesis or degradation. Some affect the liver, heart and muscle, while others are tissue-specific. Hypoglycaemia occurs in most hepatic GSDs (types I, III, VI and IX) but some type VI and IX patients only have a mild predisposition. Hepatomegaly is usually present, though it can be missed, particularly in GSD VI and IX. ${ }^{19}$ GSD 
Box 1 Differential diagnosis of hypoglycaemia in childhood

- Idiopathic ketotic hypoglycaemia

- Hyperinsulinaemic hypoglycaemia

- Exogenous insulin (diabetes, factitious hyperinsulinism)

- Congenital hyperinsulinism

- Dumping syndrome

- Insulinoma

- Beckwith-Wiedemann syndrome

- Adrenal insufficiency

- Primary adrenal insufficiency

- Withdrawal of exogenous corticosteroids

- Hypopituitarism

- Growth hormone deficiency

- Hypopituitarism

- Isolated growth hormone deficiency

- Glycogenoses

- Glycogen storage diseases (I, III, VI, IX)

- Glycogen synthase deficiency (GSD 0)

- Glucose transporter defects

- Fanconi-Bickel syndrome

- Gluconeogenesis disorders

- Fructose-1,6-bisphosphatase deficiency

- Glucose-6-phosphatase deficiency (GSD I)

- Fatty acid oxidation disorders

- Primary carnitine deficiency and carnitine cycle defects

- Medium chain acyl-CoA dehydrogenase deficiency and other B-oxidation disorders

- Multiple acyl-CoA dehydrogenase deficiency

- Ketogenesis disorders

- HMG-CoA synthase and lyase deficiencies

- Hereditary fructose intolerance

- Branched chain organic acidaemias and maple syrup urine disease

- Mitochondrial respiratory chain defects

- Liver disease

- Infections

- Gastroenteritis

- Septicaemia

- Malaria

- Drugs (especially insulin, sulfonylureas, $\beta$-blockers, alcohol)

I is the most severe of these disorders as the deficient enzyme, glucose-6-phosphatase, is needed for the hepatic export of glucose derived from glycogenolysis and gluconeogenesis. GSD I presents in infancy as the interval between feeds is extended; hypoglycaemia is accompanied by lactic acidaemia (due to impaired gluconeogenesis) and there is usually hyperuricaemia and hypertriglyceridaemia. In other types of GSD, hypoglycaemia is accompanied by ketosis rather than lactic acidaemia.

In glycogen synthase deficiency (GSD 0) fasting leads to ketotic hypoglycaemia, with postprandial hyperglycaemia and hyperlactataemia, without hepatomegaly. Fanconi-Bickel syndrome is caused by a defect of the glucose transporter, GLUT2, but resembles GSD I; patients present in infancy with hypoglycaemia, hepatomegaly and Fanconi-type nephropathy. ${ }^{20}$

\section{Defects of gluconeogenesis}

In fructose-1,6-bisphosphatase deficiency, glucose formation from all gluconeogenic substrates is impaired. Hypoglycaemia occurs once dietary glucose and glycogen reserves are exhausted, and gluconeogenic substrates accumulate leading to lactic acidosis. Hepatomegaly may be present during episodes of acute decompensation.

\section{Inhibition of gluconeogenesis and glycogenolysis}

Hereditary fructose intolerance usually presents with gastrointestinal symptoms but transient hypoglycaemia may occur after fructose ingestion due to inhibition of gluconeogenesis and glycogenolysis by fructose-1-phosphate. Inhibition of gluconeogenesis can also lead to hypoglycaemia in the branched chain organic acidaemias (methylmalonic, propionic and isovaleric acidaemias and maple syrup urine disease).

\section{Defects of fatty acid oxidation and ketogenesis}

FAODs and defects of ketogenesis often present with hypoketotic hypoglycaemia-in the newborn period, during intercurrent illness or after prolonged fasting. The hypoglycaemia probably results from excessive glucose consumption when fat and/or ketone bodies cannot be used. In FAODs there is relative hypoketosis as fatty acid oxidation normally provides acetyl-CoA for ketogenesis. Cardiomyopathy or myopathy may also be prominent features in some FAODs. Medium chain acyl-CoA dehydrogenase deficiency (MCADD) is the most common of these disorders, with an incidence of 1:10 000 live births. ${ }^{21}$ Newborn screening for MCADD was introduced in the UK in 2009 and some other countries screen for additional FAODs.

\section{Liver disease}

Fasting hypoglycaemia occurs in acute liver failure and in infants with end-stage chronic liver disease. It results from glycogen depletion and impaired gluconeogenesis but may be exacerbated by hyperinsulinism associated with high glucose feeds. Glucose infusion rates as high as $10-15 \mathrm{mg} / \mathrm{kg} / \mathrm{min}$ may be required to maintain euglycaemia. ${ }^{22}$ Hyperinsulinaemic hypoglycaemia is relatively common in untreated tyrosinaemia type 1. Hypoglycaemia also occurs in mitochondrial disorders with liver involvement, particularly in MPV17 defects. ${ }^{23}$ It is less common in galactosaemia but can occur after a galactose load.

\section{Idiopathic ketotic hypoglycaemia}

$\mathrm{IKH}$ is the most common cause of hypoglycaemia in young nondiabetic children. Problems are usually precipitated by an intercurrent illness associated with anorexia or vomiting. These patients have no specific endocrine or metabolic defect and the diagnosis can only be made after thorough investigation to exclude recognised disorders. Adrenal insufficiency, GSD 0, VI and IX, fructose bisphosphatase deficiency and MCADD cause hypoglycaemia with ketosis and have all been misdiagnosed as IKH. Some children with IKH may have endocrine or metabolic disorders that are currently unrecognised but most cases are thought to represent the 'lower tail of the Gaussian distribution of fasting tolerance in children'. ${ }^{24}$ They may have polymorphisms or partial deficiencies affecting one or more enzymes involved in glucose homeostasis. ${ }^{25}$ Hepatic glucose production is often decreased, ${ }^{26}$ possibly due to a limited supply of the gluconeogenic precursor alanine. ${ }^{24}$

The severity of the predisposition varies; a few children become hypoglycaemic after an overnight fast when they are healthy, whereas others only have problems after gastroenteritis with prolonged vomiting. Occasionally the hypoglycaemia is associated with seizures. Problems diminish with age and, in most patients, hypoglycaemic episodes cease by about 7 years of age. 


\section{Infections}

Infections are often responsible for precipitating hypoglycaemia in patients who are predisposed, for example, by an IEM or a tendency to IKH. Anorexia and vomiting accompany many infections, particularly gastroenteritis, and the lack of food can trigger hypoglycaemia. Moreover, there are other reasons for hypoglycaemia during infections. Glucose consumption increases due to the increased metabolic rate and there is evidence for impaired gluconeogenesis during septicaemia. ${ }^{27}$ Hypoglycaemia is common in patients with severe malaria; reduced food intake is a major factor but increased metabolic rate, impaired gluconeogenesis, glucose consumption by the malarial parasites and quinine-induced insulin secretion may all contribute. $^{28}$

\section{INVESTIGATION OF HYPOGLYCAEMIA}

Given the wide differential diagnosis, the investigation of hypoglycaemic episodes can be challenging. In many children no underlying condition is identified despite careful clinical assessment and extensive laboratory investigations.

The history may provide clues to the diagnosis. A high glucose requirement $(>8 \mathrm{mg} / \mathrm{kg} / \mathrm{min})$ to maintain normoglycaemia strongly suggests hyperinsulinism. ${ }^{17}$ The timing of hypoglycaemia is another important clue. Hypoglycaemia within a few hours of feeding suggests hyperinsulinism, though it is occasionally seen in mitochondrial defects (eg, MPV17) and after fructose ingestion in hereditary fructose intolerance. In GSD I, hypoglycaemia occurs after a brief fast (1.5-4 h), once exogenous sources of glucose are exhausted. ${ }^{29}$ Hypoglycaemia occurs later in other GSDs (4-12 h), in disorders of gluconeogenesis $(8-16 \mathrm{~h})$ and in FAODs $(>10 \mathrm{~h})$, though it can occur sooner during illness or in young patients.

The examination findings sometimes indicate the diagnoses. Hyperpigmentation may be a sign of adrenal insufficiency and persistent hepatomegaly raises the suspicion of a GSD. The liver is enlarged during the acute episode in FAODs and defects of gluconeogenesis but subsequently it usually returns to normal. Myopathy and cardiomyopathy accompany hypoglycaemia in some FAODs. In most cases, however, there are few diagnostic clues and diagnosis depends on laboratory investigations.

Box 2 Laboratory investigation of hypoglycaemic episodes

Should be taken prior to correction of glucose

- Glucose

- Lactate

- Insulin and C-peptide

- 3-hydroxybutyrate

- Free fatty acids

- Cortisol

- Growth hormone

- May be taken after correction of glucose

- Plasma or blood spot acylcarnitines

- Plasma amino acids

- Ammonia

- Urea and electrolytes

- Liver function tests

- First urine passed after episode

- Ketone bodies

- Organic acids

\section{Laboratory investigation of hypoglycaemia}

Collection of samples prior to correction of hypoglycaemia is valuable, but investigations are often omitted or samples are inappropriately handled. Box 2 lists suggested samples to be collected during and after a hypoglycaemic episode. ${ }^{8}$ The use of pre-prepared 'hypopacks' may improve sample collection. ${ }^{30}$ Treatment should not be delayed, however, as most disorders can be diagnosed by other techniques if samples are missed. If the volume of blood is limited, glucose and insulin are the most important samples to collect during an episode; it is also worth remembering to send the first urine after glucose correction for ketone body and organic acid analysis. Point-of-care testing for blood 3-hydroxybutyrate is now widely available. For glucose, laboratory measurement is essential as point-of -care testing can be unreliable at low blood glucose concentrations.

Detectable insulin levels during hypoglycaemia are consistent with hyperinsulinism, but may not always be demonstrated as insulin has a short half-life (5-6 min) in the circulation. A single sample may miss rapid release, while hepatic clearance of insulin may give falsely low peripheral blood levels. ${ }^{31}$ Additional corroborative evidence suggesting hyperinsulinism includes suppression of both free fatty acids and 3hydroxybutyrate, and an increase in blood glucose $(>1.5 \mathrm{mmol} / \mathrm{L})$ in response to glucagon or octreotide. Increased insulin concentrations without corresponding increases in C-peptide suggest exogenous insulin administration and may be part of a fabricated and induced illness scenario.

The absence of ketone bodies on urinalysis may suggest hyperinsulinism, an FAOD or, rarely, a defect of ketone body synthesis. Importantly, the presence of ketone bodies ${ }^{32}$ does not exclude an FAOD and acylcarnitine analysis must be undertaken. The concentrations of free fatty acids and ketone bodies rise simultaneously during fasting in normal individuals and the logarithms of their values are linearly related. ${ }^{33}$ Patients with FAODs and ketogenesis defects show relative hypoketosis with a low 3-hydroxybutyrate:free fatty acids ratio.

Inclusion of growth hormone and cortisol in the hypoglycaemia screen is controversial. Growth hormone and cortisol levels are frequently below normal reference ranges in children undergoing fasting tests ${ }^{34}$ and do not necessarily correlate with the degree of hypoglycaemia when measured as part of a hypoglycaemia screen. ${ }^{35}$ If cortisol deficiency is suspected, the diagnosis is established by an ACTH challenge test (Synacthen test). In suspected growth hormone deficiency, magnetic resonance pituitary scanning to visualise anatomical defects in hypothalamic-pituitary axis and growth hormone dynamic stimulation testing is required in association with careful assessment of growth.

Though it is helpful to have acute samples, many causes of hypoglycaemia can now be diagnosed without these. Most FAODs that cause hypoglycaemia can be identified by acylcarnitine analysis on blood obtained when the patient is well. If the clinical features suggest a GSD, glycogen synthase deficiency or a defect of gluconeogenesis, the diagnosis may now be confirmed by sequencing a 'panel' of relevant genes. The results of genetic tests must, however, be interpreted carefully, as mutations may not always be identified and variants of uncertain significance are likely to be found.

In the past, diagnostic fasts were undertaken routinely if appropriate samples had not been obtained at the time of hypoglycaemia. ${ }^{33}$ This is now undertaken much less often, unless $\mathrm{CHI}$ is suspected. Prolonged diagnostic fasting tests are hazardous and require close monitoring on an experienced unit. ${ }^{33}$ 
Moreover, the diagnostic yield is low as many conditions are now diagnosed by alternative techniques, such as those listed above. Thus, fasting is now more often undertaken following diagnosis to monitor the response to treatment or to establish the fasting tolerance and plan feeding regimens for children with disorders such as GSD, CHI or severe cases of IKH.

\section{MANAGEMENT OF HYPOGLYCAEMIA}

Correction of hypoglycaemia should follow standard practice guidelines, using oral glucose (in the conscious patient), intravenous glucose or glucagon injection (box 3). In most cases, euglycaemia can be maintained with a glucose infusion rate of $4-8 \mathrm{mg} / \mathrm{kg} / \mathrm{min}$, but in suspected CHI, rates of up to $10-20 \mathrm{mg} /$ $\mathrm{kg} / \mathrm{min}$ may be required, sometimes necessitating a central venous catheter.

\section{Management of hyperinsulinism}

The initial management of CHI is additional glucose supplementation to achieve euglycaemia. Glucagon infusions may reduce the requirement for large amounts of glucose but the long-term strategy is to reduce unregulated insulin secretion. First-line therapy is with the $\mathrm{K}_{\mathrm{ATP}}$ channel opening agent diazoxide. Patients who do not respond to diazoxide may respond to the somatostatin analogue, octreotide but the efficacy of this is often limited by tachyphylaxis. Subtotal pancreatectomy may be required if medical therapy fails but it usually leads to diabetes later in life. In contrast, the surgical treatment for focal $\mathrm{CHI}$ is a lesionectomy, which is curative and without a risk of developing diabetes.

\section{Management of other endocrine disorders}

The treatment of adrenal insufficiency is by oral replacement of cortisol by hydrocortisone and where necessary, aldosterone replacement by fludrocortisone. During illness, hydrocortisone replacement should be doubled, mimicking increased physiological requirements. Management of hypoglycaemia during an adrenal crisis requires intramuscular or intravenous bolus doses of hydrocortisone in addition to glucose replacement.

In children experiencing hypoglycaemia from severe growth hormone deficiency, daily subcutaneous recombinant growth hormone usually ameliorates hypoglycaemia over a few days.

\section{Box 3 Emergency management of hypoglycaemia}

If patient is fully conscious and not vomiting, give oral glucose 10-20 g, for example, $200 \mathrm{~mL}$ milk, $100 \mathrm{~mL}$ Lucozade (original) or 2 teaspoons sugar, followed by a snack of starchy carbohydrates or a milk feed in infants

In patients with altered consciousness:

1. Establish intravenous access

2. Give $2 \mathrm{~mL} / \mathrm{kg} 10 \%$ glucose as intravenous bolus

3. Follow with infusion containing $10 \%$ glucose, for example, $0.45 \% \mathrm{NaCl}+10 \%$ glucose at standard maintenance rates (adjusted to maintain a blood glucose concentration of 4$6 \mathrm{mmol} / \mathrm{L})$

If intravenous glucose cannot be given, use

- buccal dextrose gel and/or

- intramuscular glucagon:

- $500 \mu \mathrm{g}$ for patients $<25 \mathrm{~kg}$

- $1 \mathrm{mg}$ for patients $>25 \mathrm{~kg}$
For children with diabetes in the UK, treatment is recommended if the blood glucose concentration is less than $4.0 \mathrm{mmol} / \mathrm{L}$. Hypoglycaemia should be prevented by frequent blood glucose monitoring, flexible insulin regimens and individualised glycaemic targets. ${ }^{36}$ Systems that deliver both insulin and glucagon through an automated subcutaneous pump promise to reduce the risk of hypoglycaemia.

\section{Management of hypoglycaemia in IKH and IEMs}

The measures to prevent hypoglycaemia are broadly similar for IKH and most IEMs (though there may also be disease-specific treatment, such as a low-fat diet and medium chain triglycerides in some long chain FAODs). Families are advised to give frequent feeds and avoid prolonged periods of fasting. The length of the fasting period tolerated will vary between individuals and depends on the underlying condition. Children with MCADD aged over 1 year can safely tolerate an overnight fast of $12 \mathrm{~h}$ when they are well. In contrast, children with GSD I may have a fasting tolerance as short as $1-1.5 \mathrm{~h}$ and usually require continuous enteral feeds overnight.

Uncooked corn starch is digested slowly and can therefore be used to increase the interval between feeds in patients with a short fasting tolerance, such as those with GSD I. It is seldom tolerated before 1 year of age due to a lack of pancreatic amylase but, subsequently, regular daytime doses are used in many patients with GSDs. Night-time doses can prevent the need for continuous overnight feeds in some patients with GSDs and they are also useful for patients with other conditions who cannot tolerate overnight fasting.

The fasting tolerance is shorter during infections in many IEMs. As well as increasing the tendency to hypoglycaemia, catabolism often leads to the accumulation of toxic metabolites. It is, therefore, important to minimise catabolism, primarily by maintaining a high glucose intake with additional measures in specific disorders. Most illnesses are managed at home with an 'emergency regimen' of drinks containing soluble glucose polymer to provide large amounts of carbohydrate and calories. ${ }^{37}$ If children are unable to tolerate the oral emergency regimen, they are admitted to hospital for intravenous glucose. Patients should receive this treatment even if their blood glucose is normal as its primary purpose is to reduce catabolism and prevent problems. Patients with fatty acid oxidation defects, for example, can develop arrhythmias or encephalopathy without hypoglycaemia, presumably due to accumulation of toxic metabolites. ${ }^{38}$ If it occurs, hypoglycaemia is a relatively late feature.

\section{CONCLUDING REMARKS}

Hypoglycaemia is a common clinical problem in children. The differential diagnoses and acute management have remained similar for many years, though new causes of hyperinsulinism have been recognised. It remains extremely helpful to collect samples during acute hypoglycaemia but diagnostic fasts are now performed much less frequently and other techniques, such as molecular genetics, have become more important over the past decade.

Contributors AG wrote the first draft and IB provided additional information on endocrine disorders as well as critical comments. AAMM provided additional information and critical comments. All authors have read and reviewed the final manuscript.

Competing interests None declared. 


\section{REFERENCES}

1 Kanaka-Gantenbein C. Hypoglycemia in childhood: long-term effects. Pediatr Endocrinol Rev 2004;1(Suppl. 3):530-6.

2 Stanley CA, Rozance PJ, Thornton PS, et al. Re-evaluating "transitional neonatal hypoglycemia": mechanism and implications for management. J Pediatr 2015:166:1520-5.e1.

3 Güemes M, Rahman SA, Hussain K. What is a normal blood glucose? Arch Dis Child 2016:101:568-73.

4 Wilcox G. Insulin and Insulin Resistance. Clin Biochem Rev 2005;26:19-39.

5 Saudubray JM, De Lonlay P, Touati G, et al. Genetic hypoglycaemia in infancy and childhood: pathophysiology and diagnosis. J Inherit Metab Dis 2000:23:197-214.

6 Sprague JE, Arbelaez AM. Glucose counterregulatory responses to hypoglycemia. Pediatr Endocrinol Rev 2011;9:463-73; quiz 74-5.

7 Cryer PE. Hypoglycemia, functional brain failure, and brain death. J Clin Invest 2007;117:868-70.

8 National Metabolic Biochemistry Network. Best Practice Guidelines for the Investigation of Hypoglycaemia in Children. 2012. http://www.metbio.net/ metbioGuidelines.asp (accessed 8 May 2015).

9 Josefson J, Zimmerman D. Hypoglycemia in the Emergency Department. Clin Pediat Emerg Med 2009;10:285-91.

10 Morris AA. Cerebral ketone body metabolism. J Inherit Metab Dis 2005;28:109-21.

11 Fernandes J, Berger R, Smit GP. Lactate as a cerebral metabolic fuel for glucose-6-phosphatase deficient children. Pediatr Res 1984;18:335-9.

12 Boyle PJ, Schwartz NS, Shah SD, et al. Plasma glucose concentrations at the onset of hypoglycemic symptoms in patients with poorly controlled diabetes and in nondiabetics. N Engl J Med 1988;318:1487-92.

13 Cryer PE. Mechanisms of hypoglycemia-associated autonomic failure in diabetes. N Engl J Med 2013;369:362-72

14 Banarer S, Cryer PE. Sleep-related hypoglycemia-associated autonomic failure in type 1 diabetes: reduced awakening from sleep during hypoglycemia. Diabetes 2003:52:1195-203.

15 Thornton PS, Stanley CA, De Leon DD, et al. Recommendations from the Pediatric Endocrine Society for Evaluation and Management of Persistent Hypoglycemia in Neonates, Infants, and Children. J Pediatr 2015;167:238-45.

16 Arnoux JB, Verkarre V, Saint-Martin C, et al. Congenital hyperinsulinism: current trends in diagnosis and therapy. Orphanet J Rare Dis 2011;6:63.

17 Kapoor RR, Flanagan SE, James C, et al. Hyperinsulinaemic hypoglycaemia. Arch Dis Child 2009:94:450-7.

18 Bufler P, Ehringhaus C, Koletzko S. Dumping syndrome: a common problem following Nissen fundoplication in young children. Pediatr Surg Int 2001:17:351-5.

19 Brown L, Corrado M, van der Ende R, et al. Evaluation of glycogen storage disease as a cause of ketotic hypoglycemia in children. J Inherit Metab Dis 2015;38:489-93.
20 Santer R, Steinmann B, Schaub J. Fanconi-Bickel syndrome-a congenital defect of facilitative glucose transport. Curr Mol Med 2002;2:213-27.

21 Oerton J, Khalid JM, Besley G, et al. Newborn screening for medium chain acyl-CoA dehydrogenase deficiency in England: prevalence, predictive value and test validity based on 1.5 million screened babies. J Med Screen 2011;18:173-81.

22 Squires RH, Alonso EM. Liver disease in children. 4th edn. Cambridge University Press, 2014:46.

23 Parini R, Furlan F, Notarangelo L, et al. Glucose metabolism and diet-based prevention of liver dysfunction in MPV17 mutant patients. J Hepatol 2009;50:215-21.

24 Huidekoper $H$, Duran $M$, Turkenburg $M$, et al. Fasting adaptation in idiopathic ketotic hypoglycemia: a mismatch between glucose production and demand. Eur J Pediatr 2008:167:859-65.

25 Lang TF, Hussain K. Chapter six-Pediatric hypoglycemia. In: Gregory SM, ed. Advances in clinical chemistry. Elsevier, 2014:211-45.

26 Bodamer OA, Hussein K, Morris AA, et al. Glucose and leucine kinetics in idiopathic ketotic hypoglycaemia. Arch Dis Child 2006;91:483-6.

27 Goto M, Zeller WP, Lichtenberg RC. Decreased gluconeogenesis and increased glucose disposal without hyperinsulinemia in 10-day-old rats with endotoxic shock. Metabolism 1994;43:1248-54.

28 Madrid L, Lanaspa M, Maculuve SA, et al. Malaria-associated hypoglycaemia in children. Expert Rev Anti Infect Ther 2015;13:267-77.

29 Laforet $P$, Weinstein $D$, Smit $P$. The glycogen storage diseases and related disorders. In: Saudubray JM, van den Berghe G, Walter J, eds. Inborn metabolic diseases. 5th edn. Heidelberg: Springer-Verlag, 2012:117-39.

30 Lang TF, Cardy D, Carson D, et al. Audit of acute hypoglycaemia in children: re-audit of procedures for diagnosis. Ann Clin Biochem 2008;45(Pt 5):486-8.

31 Palladino AA, Bennett MJ, Stanley CA. Hyperinsulinism in infancy and childhood: when an insulin level is not always enough. Clin Chem 2008;54:256-63.

32 Fletcher JM, Pitt JJ. Fasting medium chain acyl-coenzyme A dehydrogenasedeficient children can make ketones. Metabolism 2001;50:161-5.

33 Morris AA, Thekekara A, Wilks Z, et al. Evaluation of fasts for investigating hypoglycaemia or suspected metabolic disease. Arch Dis Child 1996;75:115-19.

34 Kelly A, Tang R, Becker S, et al. Poor specificity of low growth hormone and cortisol levels during fasting hypoglycemia for the diagnoses of growth hormone deficiency and adrenal insufficiency. Pediatrics 2008;122:e522-8.

35 Elder CJ, Wright VJ, Wright NP. Time to end the routine testing of growth hormone and cortisol on hypoglycaemia screens? Arch Dis Child 2009;94:1000-1.

36 Cryer PE. Hypoglycemia-associated autonomic failure in diabetes. Handb Clin Neurol 2013;117:295-307.

37 Dixon MA, Leonard JV. Intercurrent illness in inborn errors of intermediary metabolism. Arch Dis Child 1992;67:1387-91.

38 Mayell SJ, Edwards L, Reynolds FE, et al. Late presentation of medium-chain acyl-CoA dehydrogenase deficiency. J Inherit Metab Dis 2007;30:104. 